BULL. AUSTRAL. MATH. SOC.

\title{
UNIVALENT FUNCTIONS WITH UNIVALENT GELFOAD-LEONTEV DERIVATIVES
}

\author{
O.P. JUNEJA AND S.M. SHAH
}

Let $\left\{d_{n}\right\}_{1}^{\infty}$ be a nondecreasing sequence of positive numbers. We consider Gelfond-Leontev derivative $D f(z)$, of a function $f(z)=\sum_{n=0}^{\infty} a_{n} z^{n},|z|<R$, defined by $D f(z)=\sum_{n=1}^{\infty} d_{n} a_{n} z^{n-1}$, for univalence and growth properties, and extend some results of Shah and Trimble. Set $e_{n}=\left(d_{1} d_{2} \ldots d_{n}\right)^{-1}, n \geq 1, e_{0}=1$, $p(z)=\sum_{n=0}^{\infty} e_{n} z^{n}$. Let $r$ be the radius of convergence of $p(z)$. We state parts of Theorem $I$ and Corollaries. Let $f$ and all $D^{k} f, k=1,2, \ldots$, be analytic and univalent in the unit disk $U$. Then
(i) $|f(z)| \leq\left|a_{0}\right|+\left(\left|a_{1}\right| d_{1} / 2 d_{2}\right)\left\{p\left(2 d_{2}|z|\right)-1\right\},|z|<r / 2 d_{2}$,
(ii) $\left|D^{k} f(z)\right| \equiv\left|\sum_{n=k}^{\infty}\left(e_{n-k} / e_{n}\right) a_{n} z^{n-k}\right| \leq\left|a_{1}\right| d_{1}\left(2 d_{2}\right)^{k-1} p\left(2 d_{2}|z|\right)$, $k \geq 1$,
(iii) if $p$ is entire and of growth $(\rho, T)$ then $f$ must be entire and of growth not exceeding $\left(\rho, 2 d_{2} T\right)$,

Received 30 November 1983.

Copyright Clearance Centre, Inc. Serial-fee code: 0004-9727/84 $\$ A 2.00+0.00$. 
(iv) if $D$ corresponds to the shift operator $\left(d_{n} \equiv 1\right)$, then

$$
|f(z)|=0(1-2|z|)^{-1} \text { as } z \rightarrow \frac{1}{2} \text {. }
$$

Another class of functions is defined by a condition of the form $\left|a_{n+1}\right| a_{n} \mid \leq b_{n+1} / d_{n+1}$, where $\left\{b_{n}\right\}_{1}^{\infty}$ is a sequence of positive numbers satisfying an inequality, and it is shown that all functions in this class along with all their Gelfond-Leontev successive derivatives are regular and univalent in $U$. An extension of the definition of a linear invariant family is given and results analogous to $(i)$ and $(i i)$ are stated.

\section{Introduction}

Let $f(z)=\sum_{n=0}^{\infty} a_{n} z^{n}$ be an analytic function in the disc $|z|<R$. Let $\left\{d_{n}\right\}_{n=1}^{\infty}$ denote a non-decreasing sequence of positive numbers and $D$ the operator which transforms the function

$$
f(z)=\sum_{n=0}^{\infty} a_{n} z^{n}
$$

into

$$
D f(z)=\sum_{n=1}^{\infty} d_{n} a_{n^{2}}{ }^{n-1}
$$

For $k=1,2, \ldots$, the $k$ th iterate of $D$ is given by (1.3) $\quad D^{k} f(z)=\sum_{n=k}^{\infty} d_{n} \cdots d_{n-k+1} a_{n} z^{n-k}=\sum_{n=k}^{\infty} \frac{e_{n-k}}{e_{n}} a_{n} z^{n-k}$

where $e_{0}=1$ and $e_{n}=\left(d_{1} d_{2} \ldots d_{n}\right)^{-1}, n=1,2, \ldots$. If $d_{n} \equiv n$, $D$ corresponds to the ordinary derivative whereas if $d_{n} \equiv 1, D$ corresponds to the shift operator $S^{*}$ which transforms

$$
f(z)=\sum_{n=0}^{\infty} a_{n} z^{n} \text { into } S^{*} f(z)=\sum_{n=1}^{\infty} a_{n} z^{n-1}
$$


The operator $D$ is called the Gelfond-Leontev derivative [7] of $f$. The operators $D$ have been investigated extensively by Kazmin [9], Buckholtz and Frank [3, 4] and others.

Set

$$
p(z)=\sum_{n=0}^{\infty} e_{n} z^{n}
$$

It is clear that $p(0)=1$ and $D p(z)=p(z)$. Thus $p(z)$ bears the same relationship to the operator $D$ which the exponential function bears to the ordinary differentiation. If $r$ be the radius of convergence of $p(z)$ then we have

$$
r=\lim _{n \rightarrow \infty} d_{n}=\sup _{1 \leq n<\infty} d_{n}
$$

Define the $p$-type of the function $f(z)=\sum_{n=0}^{\infty} a_{n} z^{n}$ to be the number

$$
\tau_{p}(f)=\underset{n \rightarrow \infty}{\lim \sup }\left|a_{n} / e_{n}\right|^{1 / n} \text {. }
$$

If $r<\infty$, it is easy to check that

$$
\tau_{p}(f)=r / R \text {. }
$$

If $p(z)$ is entire, p-type is a growth measure introduced by Nachbin [2, p. 6] and [11] which can be related to the maximum modulus of $f$. Further, for $p(z)$ entire, $\tau_{p}(f)<\infty$ implies that $f$ is entire.

Shah and Trimble have, in a series of papers (see, for example, [17] to [22]), studied properties of functions $f$ such that $f$ and its successive ordinary derivatives are univalent in the unit disc $U$. They showed that such an $f$ must be an entire function of exponential type. In the present paper we consider functions $f$ such that $f$ along with its Gelfond-Leontev derivatives is univalent in $U$ and show that such an $f$ must be of finite $p$-type. We shall suppose throughout that the operator $D$ is defined by (1.2) and that $p(z)$, given by (1.5), has radius of convergence $r \quad(0<r \leq \infty)$. 


\section{The class $E(D)$}

Let $S$ denote, as usual, the family of functions $h$ of the form

$$
h(z)=z+\sum_{n=2}^{\infty} b_{n} z^{n}
$$

which are analytic and univalent in the unit disc $U$. It is well known (see, for example, [13, p. 20]) that

$$
\left|b_{2}\right| \leq 2
$$

and

$$
\left|b_{3}-b_{2}^{2}\right| \leq 1
$$

both the inequalities being sharp.

It is further known [13, p. 44] that if $h$, defined by (2.1), is analytic in $U$ and if

$$
\sum_{n=2}^{\infty} n\left|a_{n}\right| \leq 1
$$

then $h$ is univalent in $U$, that is, $h \in S$. This condition (2.4), on the moduli of the coefficients alone, is best possible in the sense that if (2.4) does not hold then the arguments of the coefficients can be so altered that the new function defined by

$$
z+\sum_{2}^{\infty} a_{n} z^{n}
$$

is no longer univalent in $U$ (see [8], [10]).

Let $E(D)$ denote the family of functions of the form (2.1) such that $f$ and all its Gelfond-Leontev derivatives $D^{k_{f}}$ are analytic and univalent in $U$. Note that when $d_{n} \equiv n, D$ corresponds to the ordinary derivative and $E(D)$ is then the class $E$ considered by Shah and Trimble [17].

THEOREM 1. Let $f$ represented by (1.1) be such that $f$ and all $D^{k} f$ are analytic and ronivalent in $U$; then 
(2.5) $f$ is of finite p-type not exceeding $2 d_{2}$,

(2.6) $|f(z)| \leq\left|a_{0}\right|+\left(\left|a_{1}\right| d_{1} / 2 d_{2}\right)\left|p\left(2 d_{2}|z|\right)-1\right|,|z|<r / 2 d_{2}$,

(2.7) $\left|b^{k} f(z)\right| \leq\left|a_{1}\right| d_{1}\left(2 d_{2}\right)^{k-1} p\left(2 d_{2}|z|\right), k \geq 1$,

(2.8) $E(D)$ is a normal family in $|z|<t<r / 2 d_{2}$ for all $t$ satisfying $0<t<r / 2 d_{2}$.

Proof. Since, for $k=1,2, \ldots$,

$$
D^{k} f(z)=\sum_{n=k}^{\infty} d_{n} \cdots d_{n-k+1} a_{n} z^{n-k}
$$

is univalent in $U$, it follows that $a_{k+1} \neq 0$. Define $H_{k}$ in $U$ by

$$
H_{k}(z)=\frac{D^{k} f(z)-D^{k} f(0)}{d_{2} \cdots d_{k+1} a_{k+1}}=z+\frac{a_{k+2}}{d_{2}} \frac{a_{k+2}}{a_{k+1}} z^{2}+\ldots .
$$

It is clear that $H_{k} \in S$. By (2.2), we have, therefore,

$$
\left|\frac{a_{k+2}}{d_{2}} \cdot \frac{a_{k+2}}{a_{k+1}}\right| \leq 2, k=1,2, \ldots .
$$

An induction process gives

$$
\left|a_{k}\right| \leq e_{k}\left(2 d_{2}\right)^{k-1} d_{1}\left|a_{1}\right|, k=1,2, \ldots .
$$

Thus

$$
\underset{k \rightarrow \infty}{\operatorname{Iim} \sup }\left|\frac{a_{k}}{e_{k}}\right|^{1 / k} \leq 2 d_{2}
$$

showing that $f$ is of finite p-type not exceeding $2 d_{2}$.

Using the estimates (2.10) in the relations

$$
|f(z)| \leq \sum_{n=0}^{\infty}\left|a_{n}\right||z|^{n}
$$

and 


$$
\left|D^{k} f(z)\right| \leq \sum_{n=k}^{\infty} \frac{e_{n-k}}{e_{n}}\left|a_{n}\right||z|^{n-k}
$$

easily leads to (2.6) and (2.7). The assertion (2.8) is a consequence of local boundedness of $E(D)$ obtained from (2.6). Hence the theorem.

COROLLARY 1. If $p$ is entire, then $f \in E(D)$ must be entire and relations (2.6) and (2.7) hold for all $z \in \mathbb{C}$. Assertion (2.8) is valid on every compact subset of $\mathbb{C}$.

COROLLARY 2. If $p$ is an entire function of growth $(\rho, T)$ (ef. $[1, \mathrm{p} .8])$ then $f \in E(D)$ must be an entire fronction of growth not exceeding $\left(\rho, 2 d_{2} T\right)$.

It is known that if $f$ is analytic in $|z|<R(<\infty)$, its order $\rho_{0}$ is defined by (see, for example, [23])

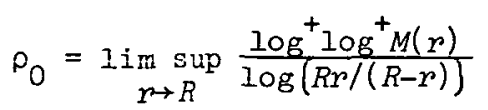

where $M(r)=\max _{|z|=r}|f(z)|$. Taking this into consideration, we have

COROLLARY 3. If $D$ corresponds to the shift operator $S^{*}$ defined by (1.4), then, by (2.6), $|f(z)|=O(1-2|z|)^{-1}$ as $|z|+\frac{1}{2}$ and $f \in E(D)$ must be of zero order in $|z|<\frac{1}{2}$.

REMARK. Theorem 1 could also be modelled in terms of "admissible property" as done in Theorem $I$ of [19], thereby generalizing that theorem.

\section{Functions in the class $S$}

A function $f \in S$ may have $f^{\prime}$ univalent in $U$ but its GelfondLeontev derivative $D f$ may not be univalent in $U$. To see this, let

$$
f(z)=z+\sum_{n=2}^{\infty} a_{n} z^{n}
$$

be such that $a_{n}$ is negative for $n \geq 3, a_{2}=4 /(5+8 \log 2)$, $\left|a_{n}\right|=a_{2} /\left(2^{n-3} \cdot n(n-1)\right)$. Using (2.4), it is easy to check that $f^{\prime}$ is univalent in $U$. However, if we take $d_{n}$ to be a non-decreasing sequence 
in which $d_{3}=3 d_{2}$, then $D f$ is seen not to be univalent in $U$. In fact, it is possible to have such an $f$ with p-type as large as we please. This is demonstrated by the following

THEOREM 2. There exists an $f \in S$ such that $f^{\prime}$ is vonivalent in $U$; its Gelfond-Leontev derivative $D f$ is not univalent in $U$ and p-type of $f$ is as large as we please.

Proof. Let

$$
f(z)=z+\sum_{n=2}^{\infty} a_{n} z^{n}
$$

where we choose $a_{n}=a_{2} / n(n-1) 2^{n-3}$ for $n=3,4, \ldots$ and $a_{2}>0$ such that $\sum_{n=2}^{\infty} n a_{n} \leq 1$. Then $f \in S$. Further

$$
f^{\prime}(z)=1+\sum_{n=2}^{\infty} n a_{n} z^{n-1}
$$

is easily seen to satisfy a condition of the form (2.4) and so $f^{\prime}$ is univalent in $U$. Now choose $d_{1}=1,\left\{d_{n}\right\} \uparrow$, and for some $N \geq 2$, choose nondecreasing $d_{N+1}=d_{2}(N+2) \cdot 2^{N-2}, d_{N+k}=d_{N+1}$ for all $k>1$. Then

$$
\frac{d_{N+1} a_{N+1}}{d_{2} a_{2}}=\frac{N+2}{N(N+1)}>\frac{1}{N} \text {. }
$$

Hence, by a theorem of Qin Yuan-Xun [14] there exists a real number $\varphi$ such that

$$
z+e^{i \varphi} \sum_{n=3}^{\infty} \frac{d_{n} a}{d_{2} a_{2}} z^{n-1}
$$

is not univalent in $U$. Now let

$$
F(z)=z+a_{2} z^{2}+\sum_{n=3}^{\infty} a_{n} e^{i \varphi_{z} n}
$$

Since

$$
\sum_{n=2}^{\infty} n\left|a_{n} e^{i \varphi}\right|=\sum_{n=2}^{\infty} n a_{n} \leq 1
$$


by (2.4), $F \in S$. Further, $F^{\prime}$ is also seen to be univalent in $U$. Now, by (1.2),

$$
D F(z)=d_{1}+d_{2} a_{2} z+d_{3} a_{3} e^{i \varphi} z^{2}+\ldots+d_{N+1} a_{N+1} e^{i \varphi_{2} N}+\ldots .
$$

In view of (3.2), $D F(z)$ is not univalent in $U$. Further

$$
\underset{n \rightarrow \infty}{\limsup }\left|\frac{a_{n}}{e_{n}}\right|^{1 / n}=\frac{d_{N+1}}{2} \text {. }
$$

Thus by choosing $d_{N+1}$ sufficiently large, we can have $p$-type of $F(z)$ as large as we please.

\section{Radius of univalence of $D^{n} f$}

Let $f(z)=\sum_{n=0}^{\infty} a_{n} z^{n}$ be analytic in $|z|<R$ and let $\rho_{n}$ be the

largest number with the property that $D^{n} f$ is analytic and univalent in an open disc about the origin of radius $\rho_{n}$. We now investigate the relation between the growth of $\left\{\rho_{n}\right\}$ and the radius of convergence of $f$ about the origin. We thus have

THEOREM 3. Let $f$ be defined by (1.1) with radius of convergence $R$ and let $\rho_{n}$ be the radius of univalence of $D^{n} f$. Then

$$
\underset{n \rightarrow \infty}{\lim \inf } d_{n} \rho_{n} \leq \underset{n \rightarrow \infty}{\lim \inf }\left[\prod_{i=N}^{n} \rho_{i} d_{i}\right]^{1 / n} \leq 2 d_{2} R \text {, }
$$

where $N$ denotes the smallest non-negative integer such that for $n \geq N$, $\rho_{n}>0$. Further, if $\left|a_{n} / a_{n+1}\right|$ is eventually a positive and nondecreasing sequence, then

$$
\underset{n \rightarrow \infty}{\lim \sup } d_{n} \rho_{n} \leq 2 d_{2} R
$$

in case $f$ is of finite p-type, then we also have $(4.2)^{\prime}$

$$
\lim _{n \rightarrow \infty} d_{n} \rho_{n} \leq d_{2} R \sqrt{d_{3} /\left(d_{3}-d_{2}\right)} \text {. }
$$


Proof. If $\rho_{n}=0$ for an infinity of $n,(4.1)$ is trivially true. If $\rho_{n}>0$ for $n \geq N$, then it is obvious that $a_{n+1} \neq 0$ for $n \geq N$. Let

$$
F_{n}(z):=D^{n} f\left(\rho_{n} z\right)=\frac{a_{n}}{e_{n}}+\frac{e_{1}}{e_{n+1}} a_{n+1} \rho_{n} z+\frac{e_{2}}{e_{n+2}} a_{n+2} \rho_{n}^{2_{z} z^{2}}+\ldots, \quad z \in U
$$

Then the function $k_{n}$ defined by

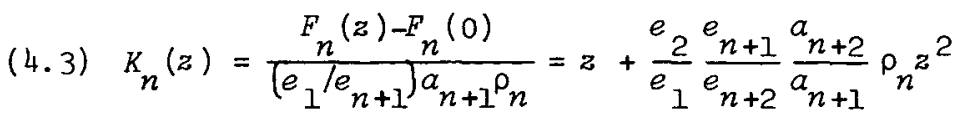

$$
+\frac{e_{3}}{e_{1}} \frac{e_{n+1}}{e_{n+3}} \frac{a_{n+3}}{a_{n+1}} \rho_{n}^{2} z^{3}+\ldots
$$

is in $S$.

Applying (2.2) we have

$$
e_{2} e_{n+1}\left|a_{n+2}\right| \rho_{n} \leq 2 e_{1} e_{n+2}\left|a_{n+1}\right| \text {, }
$$

$$
\rho_{n} d_{n+2} \leq 2 d_{2}\left|a_{n+1}\right| a_{n+2} \mid, n \geq N \text {; }
$$

that is

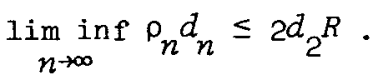

Inductive process applied to $(4.4)$ gives

$$
\prod_{k=N}^{n} \rho_{k} d_{k} \leq\left(2 d_{2}\right)^{n-N+1}\left|a_{N+1} / a_{n+2}\right|
$$

Hence

$$
\liminf _{n \rightarrow \infty}\left[\prod_{k=N}^{n} \rho_{k} d_{k}\right]^{1 / n} \leq 2 d_{2} R
$$

Since left-hand inequality of (4.1) is readily seen to be true, (4.6) gives (4.1).

If $f$ is such that $\left|a_{n} / a_{n+1}\right|$ is a positive and nondecreasing sequence then $R=\lim _{n \rightarrow \infty}\left|a_{n} / a_{n+1}\right|$ and (4.4) gives 
$(4.7)$

$$
\lim _{n \rightarrow \infty} \rho_{n} d_{n} \leq 2 d_{2} R
$$

If $R=\infty,(4.2)^{\prime}$ obviously holds. In case $R<\infty$ and $f$ is of finite p-type then, by (1.7), $r<\infty$ and so $d_{n} \sim d_{n+1}$ as $n \rightarrow \infty$.

We now apply (2.3) to the function $K_{n}$ defined by (4.3) and obtain

$$
\left|\left[\frac{d_{n+2}}{d_{2}} \frac{a_{n+2}}{a_{n+1}} \rho_{n}\right]^{2}-\frac{d_{n+3}}{d_{3}} \frac{d_{n+2}}{d_{2}} \frac{a_{n+3}}{a_{n+1}} \rho_{n}^{2}\right| \leq 1
$$

that is,

$$
\left|\frac{d_{n+2}}{d_{2}}\right|^{2}\left|\frac{a_{n+2}}{a_{n+1}}\right|^{2} \rho_{n}^{2}-\left|\frac{d_{n+3}}{d_{3}} \frac{d_{n+2}}{d_{2}}\right|\left|\frac{a_{n+3}}{a_{n+2}}\right|\left|\frac{a_{n+2}}{a_{n+1}}\right| \rho_{n}^{2} \leq 1
$$

or

$$
\lim _{n \rightarrow \infty} \sup \left(d_{n} \rho_{n}\right)^{2}\left|\frac{1}{d_{2}^{2} R^{2}}-\frac{1}{d_{1} d_{3}} \frac{1}{R^{2}}\right| \leq 1
$$

$$
\limsup _{n \rightarrow \infty} d_{n} \rho_{n} \leq d_{2} \sqrt{d_{3} /\left(d_{3}-d_{2}\right)} \dot{R}
$$

(4.8) gives (4.2)'. Hence the theorem.

COROLLARY. If $\lim _{n \rightarrow \infty} d_{n} \rho_{n}=\infty$, then $f$ is a transcendental entire fimation.

If we take $f(z)=z /(1-z)$, then $R=1$ and taking $d_{n} \equiv n$, we have $\rho_{n}=\sin (\pi /(n+1))$. Thus $(4.1)$ gives

$$
\pi \leq \liminf _{n \rightarrow \infty}\left[\prod_{i=1}^{n} i \sin \frac{\pi}{i+1}\right]^{1 / n} \leq 4
$$

\section{Entire functions of finite order}

We now obtain relations between the radii of univalence of the Gelfond-Leontev derivatives of an entire function and its growth constants namely, order, lower order, and so on. Let $f(z)=\sum_{n=0}^{\infty} a_{n} z^{n}$ be an entire 
function of order $\alpha$ and the lower order $\beta$. It is known [15] that

$$
\underset{n \rightarrow \infty}{\lim \inf } \frac{\log \left|a_{n} / a_{n+1}\right|}{\log n} \leq \frac{1}{\alpha} \leq \frac{1}{\beta} \leq \lim \sup _{n \rightarrow \infty} \frac{\log \left|a_{n} / a_{n+1}\right|}{\log n}
$$

and that equality holds at both ends of (5.1) if $\left|a_{n} / a_{n+1}\right|$ forms a nondecreasing function of $n$ for $n>N$. For $0<\alpha<\infty$, let $f(z)$ be of type $T$ and lower type $t$; then $([1, p .11],[16])$,

$$
\begin{aligned}
& e \alpha T=\limsup _{n \rightarrow \infty} n\left|a_{n}\right|^{\alpha / n}, \\
& e \alpha t \geq \liminf _{n \rightarrow \infty} n\left|a_{n}\right|^{\alpha / n},
\end{aligned}
$$

where equality holds in (5.3) if $\left|a_{n} / a_{n+1}\right|$ forms a nondecreasing function of $n$ for $n>N$. We now have

THEOREM 4. Let $f$, defined by $f(z)=\sum_{n=0}^{\infty} a_{n} z^{n}$, be a transcendental entire function of order $\alpha(0<\alpha<\infty)$, lower order $\beta$, type $T$ and Lower type $t$. Let $D$ denote the Gelfond-Leontev operator defined by (1.2) and $\rho_{n}$ the radius of univalence of $D^{n} f$. If $\theta=\lim _{n \rightarrow \infty} \inf \log d_{n} / \log n$, one has

$$
\lim _{n \rightarrow \infty} \inf \frac{\log \rho_{n}}{\log n} \leq \frac{1}{\alpha}-\theta
$$

$$
\underset{n \rightarrow \infty}{\lim \inf } \frac{\rho_{n-2} d_{n}}{n^{1 / \alpha}} \leq \underset{n \rightarrow \infty}{\lim \inf }\left[\prod_{k=N}^{n} \frac{\rho_{k-2} d_{k}}{k^{1 / \alpha}}\right]^{\alpha / n} \leq \frac{\left[2 d_{2}\right]^{\alpha}}{\alpha T},
$$

where $N$ denotes the non-negative integer such that for $n \geq N, \rho_{n}>0$. Further if $\left|a_{n} / a_{n+1}\right|$ is eventualzy a positive and nondecreasing sequence, then

$$
\lim _{n \rightarrow \infty} \sup \frac{\log \rho_{n}}{\log n} \leq \frac{1}{\beta}-\theta
$$




$$
\underset{n \rightarrow \infty}{\lim \sup }\left[\prod_{k=N}^{n} \frac{\rho_{k-2} d_{k}}{k^{1 / \alpha}}\right]^{\alpha / n} \leq \frac{\left|2 d_{2}\right|^{\alpha}}{\alpha t} .
$$

Proof. By (4.4) we have

$$
\log \left|a_{n+1}\right| a_{n+2} \mid+\log \left(2 d_{2}\right)>\log \left(\rho_{n} d_{n}\right)
$$

This, coupled with extreme left inequality of (5.1), gives

$$
\frac{1}{\alpha} \geq \underset{n \rightarrow \infty}{\lim \inf } \frac{\log \left(\rho_{n} d_{n}\right)}{\log n} \geq \underset{n \rightarrow \infty}{\lim \inf } \frac{\log \rho_{n}}{\log n}+\theta
$$

which results in $(5.4)$.

Now, by $(5.2)$,

$$
\begin{aligned}
e \alpha T & =\underset{n \rightarrow \infty}{\lim \sup } n\left|a_{n}\right|^{\alpha / n} \\
& =\underset{n \rightarrow \infty}{\lim \sup } n\left[\prod_{k=N+2}^{n}\left|a_{k} / a_{k-1}\right|\right]^{\alpha / n} \\
& =\underset{n \rightarrow \infty}{\lim \sup } n\left[\prod_{k=N+2}^{n} \frac{2 d_{2}}{\rho_{k-2} d_{k}}\right]^{\alpha / n} \\
& =\left(2 d_{2}\right)^{\alpha} \lim \sup _{n \rightarrow \infty} n\left[\prod_{k=N+2}^{n} \rho_{k-2} d_{k}\right]^{-\alpha / n}
\end{aligned}
$$

or

$$
\liminf _{n \rightarrow \infty}\left[\prod_{k=N}^{n} \frac{\rho_{k-2} d_{k}}{k^{1 / \alpha}}\right]^{\alpha / n} \leq \frac{\left(2 d_{2}\right)^{\alpha}}{\alpha T}
$$

The left-hand inequality of (5.5) being obvious, the proof of (5.5) is complete. If $\left|a_{n} / a_{n+1}\right|$ is eventually a nondecreasing sequence, then, by $(5.1)$,

$$
\frac{1}{\beta}=\lim _{n \rightarrow \infty} \frac{\log \left|a_{n} / a_{n+1}\right|}{\log n}
$$

so that, for $\varepsilon>0$,

$$
\frac{1}{\beta}+\varepsilon>\frac{\log \left|a_{n} / a_{n+1}\right|}{\log n}, n>n_{0}=n_{0}(\varepsilon) \text {. }
$$


(4.4) now gives

$$
\frac{1}{\beta} \geq \lim \sup _{n \rightarrow \infty} \frac{\log \left(\rho_{n} d_{n}\right)}{\log n} \geq \lim \sup _{n \rightarrow \infty} \frac{\log \rho_{n}}{\log n}+\theta .
$$

This easily leads to (5.6). The proof of (5.7) is similar to that of (5.5) except that one has to use the relation

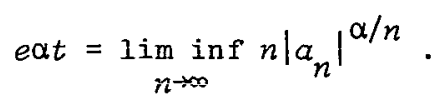

COROLLARY. (i) If $\left|a_{n} / a_{n+1}\right|$ is eventualzy a positive and nondecreasing sequence and $\beta \theta>1$ then $\lim _{n \rightarrow \infty} \rho_{n}=0$.

(ii) If $\lim _{n \rightarrow \infty} \inf d_{n} n^{-1 / \alpha}>0$ and $\lim _{n \rightarrow \infty} p_{n}=\infty$, then $T=0$.

We take $f(z)=\left(e^{\pi z}-1\right) / \pi$ and $d_{n} \equiv n$, then $\alpha=\beta=1, \theta=1$, $\rho_{n}=1$. Thus equality holds in (5.4) and (5.6).

\section{The class $E(D)$}

In the present section, we obtain a set of conditions on the coefficients of $f$ and on $\left\{d_{n}\right\}$ such that $f \in E(D)$.

Let $\left\{b_{j}\right\}_{j=1}^{\infty}$ be a sequence of positive numbers such that

(6.1) $b_{1}=1, \sum_{M=1}^{\infty} \frac{M+1}{d_{1} d_{2} \cdots d_{M+1}} \prod_{j=k+2}^{M+k+1} b_{j} \leq 1$ for $k=0,1,2, \ldots$.

Suppose that $\left\{a_{n}\right\}_{n=0}^{\infty}$ is a sequence of complex numbers such that

(6.2) $a_{0}=0, a_{1}=1$ and $\left|\frac{a_{n+1}}{a_{n}}\right| \leq \frac{b_{n+1}}{d_{n+1}}$ for $n=1,2, \ldots$.

Let $E(D)$ denote the class of functions $f$ such that

$$
f(z)=z+\sum_{n=2}^{\infty} a_{n} z^{n}
$$

satisfies condition (6.2). We now show that $E(D) \subset E(D)$. In fact we have the following 
THEOREM 5. If $f \in E(D)$, then $f$ is starlike wonivalent in $U$ and $D_{f}^{k}$ for $k=1,2, \ldots$ is univalent in $U$. Further $E(D)$ is property contained in $E(D)$.

Proof. From (1.3) we have

$$
D^{k} f(z)=\sum_{n=k}^{\infty} \frac{e_{n-k}}{e_{n}} a_{n} z^{n-k}, k=0,1,2, \ldots .
$$

By (2.4), $D^{k} f$ is univalent in $U$ if

$$
\sum_{n=2}^{\infty} \frac{d_{1} d_{2} \ldots d_{n+k}}{d_{1} d_{2} \ldots d_{n}}\left|a_{n+k}\right| n \leq \frac{d_{1} d_{2} \ldots d_{1+k}}{d_{1}}\left|a_{k+1}\right| .
$$

However (6.2) easily gives

$$
\left|a_{n+k}\right| \leq \frac{b_{k+n} \cdots b_{k+2}}{d_{k+n} \cdots d_{k+2}}\left|a_{k+1}\right| .
$$

Thus (6.4) will follow if

$$
\sum_{N=2}^{\infty} \frac{b_{k+N} \cdots b_{k+2}}{d_{N} \cdots d_{2}}\left|a_{k+1}\right| N \leq\left|a_{k+1}\right|
$$

that is,

$$
\sum_{M=1}^{\infty} \frac{(M+1)}{d_{1} d_{2} \cdots d_{M+1}} \prod_{j=k+2}^{M+k+1} b_{j} \leq 1 \text { for } k=0,1,2, \ldots \text {, }
$$

which is condition (6.1). Thus $f \in E(D)$.

To show that $E(D)$ is properly contained in $E(D)$, we take $d_{n} \equiv n$. Then $E(D)=E$ and $E(D)=E$. The function $\left(e^{\pi z}-1\right) / \pi$ belongs to $E$ but is not in $E$ (see [5]). Hence the theorem.

The class $E(D)$ may contain, in general, functions that are not entire. To see this, let

$$
d_{n}=1 \text { for } n=1,2, \ldots, b_{1}=1, b_{2}=b_{3}=\ldots=1-\frac{1}{\sqrt{2}}=x \text {, say. }
$$

Then (6.1) becomes

$$
\sum_{M=1}^{\infty}(M+1) x^{M}=\frac{1}{(1-x)^{2}}-1=1
$$


that is, (6.1) is satisfied for $k=0,1,2, \ldots$.

Let

$$
B(z)=z+\sum_{n=2}^{\infty}\left(b_{1} b_{2} \ldots b_{n}\right) z^{n}=z+\sum_{n=2}^{\infty} b_{n}^{*} z^{n}
$$

Since (6.1) and (6.2) are satisfied, $B(z)$ is in $E(D)$. However, if $R$ be the radius of convergence of $B(z)$, then

$$
R=\lim _{n \rightarrow \infty}\left|\frac{b_{n}^{*}}{b_{n+1}^{*}}\right|=\lim _{n \rightarrow \infty} \frac{1}{b_{n+1}}=\frac{\sqrt{2}}{\sqrt{2}-1}
$$

so that $B(z)$ is not entire.

Our next theorem gives conditions under which every $f \in E(D)$ is entire.

THEOREM 6. If $b_{n} / d_{n}=o(1)$ and $f \in E(D)$, then $f$ is entire.

Proof. Suppose $f(z)=z+\sum_{n=2}^{\infty} a_{n} z^{n}$ is in $E(D)$. Then, by (6.2),

$$
\left|a_{n+1} / a_{n}\right| \leq b_{n+1} / d_{n+1}=o(1)
$$

Therefore

$$
\left|a_{n}\right| a_{n+1} \mid \rightarrow \infty \text { as } n \rightarrow \infty \text {. }
$$

Thus $f$ is entire.

REMARK 1 . The condition $b_{n} / d_{n}=o(1)$ is sharp. We can construct $f \in E(D)$ such that if $b_{n} / d_{n} \neq o(1)$ then $f$ is not entire.

Suppose $\underset{n \rightarrow \infty}{\lim \sup } b_{n} / d_{n}=2 \neq 0$. We may suppose $0<2 \leq 1-1 / \sqrt{2}$.

Let $d_{n} \equiv 1, a_{n}=b_{1} \ldots b_{n}$ where we choose $\left\{b_{n}\right\}$ as follows:

$$
\begin{aligned}
b_{1} & =1, \\
b_{n} & =2 \text { if } n \text { is not a prime, } n>1, \\
& =2 / 2 \text { if } n \text { is a prime, } n>1 .
\end{aligned}
$$

If $\pi(n)$ equals the number of primes less than or equal to $n$, then 


$$
\begin{aligned}
a_{n}^{1 / n} & \sim \exp \left\{\frac{1}{n}\left(\pi(n) \log \frac{2}{2}+(n-\pi(n)) \log 2\right)\right\} \\
& \rightarrow 2 \text { as } n \rightarrow \infty \text { since } \frac{\pi(n)}{n}+0 .
\end{aligned}
$$

Further

$$
\sum_{M=1}^{\infty}(M+1) b_{k+2} \cdots b_{M+1+k} \leq \sum_{M=1}^{\infty}(M+1) z^{M}=\frac{1}{(1-2)^{2}}-1 \leq \frac{1}{[1-1+(1 / \sqrt{2})]^{2}}-1=1
$$

so that (6.1) is satisfied. Thus $f(z)=z+\sum_{n=2}^{\infty}\left(b_{1} b_{2} \cdots b_{n}\right)_{z}^{n}$ is in $E(D)$ and $f$ is not entire.

REMARK 2. There exist functions $f \in E(D)$ such that $f$ is entire and $b_{n} / d_{n} \neq o(1)$.

Let $d_{n}=1, a_{n}=b_{1} b_{2} \ldots b_{n}$ for $n \geq 1$, where we choose $\left\{b_{n}\right\}$ as follows. Let $b_{1}=1$. Let $\left\{n_{k}\right\}_{k=1}^{\infty}$ be an increasing sequence of positive integers such that $k n_{k-1}=o\left(n_{k}\right)$ and $n_{1}=10^{10}$, and

$$
\begin{aligned}
& b(n)=1 /\left(n+n_{1}\right) !, \text { if } n \neq n_{k}, n=2,3, \ldots, \\
& b\left(n_{k}\right)=L \equiv 1 / n_{1}, \text { for } k=1,2, \ldots .
\end{aligned}
$$

Let $f(z)=z+\sum_{n=2}^{\infty} a_{n} z^{n}$. Then $f \in E(D), f$ is entire and $\lim \sup b_{n} / d_{n}=L>0$. We omit the details of the proof here and also in $n+\infty$

the next

REMARK 3. Even if $b_{n} / d_{n}=o(1)$ and conditions $(6.1)$ and (6.2) are satisfied, the entire function $f(z)=z+\sum_{n=2}^{\infty} a_{n} z^{n}$ can be made to have any order $\rho(0 \leq \rho \leq \infty)$ by appropriate choice of $\left\{b_{n}\right\}$ and $\left\{d_{n}\right\}$. Thus $f(z)$ will be of infinite order if one takes $b_{n} \equiv 1$, $d_{n}=\log (n+c), n>1$, where $c$ is a positive constant such that $\log (2+c)>n \equiv 10^{10}$. Let $a_{1}=d_{1}=1, a_{n+1} / a_{n}=b_{n+1} / d_{n+1}$. 


\section{Final remarks}

Some of the work of Shah and Trimble $[17,18,19,22]$ has been extended to linear invariant families by Campbell [6]. The concept of linear invariant family was introduced by Pommerenke [12] who defined a linear invariant family to be a family of functions of the form $f(z)=z+\ldots$ which are analytic and locally univalent $\left(f^{\prime}(z) \neq 0\right)$ in $U$ such that the function

$$
\Lambda_{\varphi} f(z)=\frac{f(\varphi(z))-f(\varphi(0))}{\varphi^{\prime}(0) f^{\prime}(\varphi(0))}=z+\ldots
$$

is again a member of the family for every Mobius transformation $\varphi$ of $U$ onto $U$. If $M$ is a linear invariant family, then the order of $M$ is defined as $\alpha=\sup \left\{\left|f^{\prime \prime}(0) / 2\right|: f \in M\right\}$.

Let $u_{\alpha}$ denote the union of all linear invariant families of order at most $\alpha$. The family $u_{\alpha}$ is itself linear invariant. It is known [12] that if $\alpha<1$, then $u_{\alpha}$ is empty; $u_{1}$ is precisely the set of all normalized convex univalent functions. The class $S$ of normalized univalent functions is contained in $u_{2}$; in fact $u_{2}$ is much larger than $S$ since it contains functions of infinite valence also.

The results of the present paper can be extended for the linear invariant families $u_{\alpha}$ if instead of assuming $f$ and its (normalized) Gelfond-Leontev derivatives to be in $S$, one assumes their normalized forms to be in $u_{\alpha}$. Thus, we say that $f(z)$ has the property of $u(\alpha)$ if and only if $(f(z)-f(0)) / f^{\prime}(0)$ is in $u_{\alpha}$. Given the Gelfond-Leontev operator $D$, let $T_{\alpha}(D)$ be the set of all $f(z)=z+\ldots$ which are analytic in $U$ and for which $D^{n} f(z)$ has the property $u(\alpha)$ for all $n \geq 0$. Whatever has been obtained for the class $E(D)$ in this paper can be easily extended to the wider class $T_{\alpha}(D)$; for example Theorem 1 in this case would read as follows:

THEOREM 7. Let $f \in T_{\alpha}(D)$. Then

(i) $f$ is of finite p-type not exceeding $\alpha d_{2}$; 
(ii) $|f(z)| \leq\left(d_{1} / \alpha d_{2}\right)\left|p\left(\alpha d_{2}|z|\right)-1\right|,|z|<r / \alpha d_{2}$;

(iii) $\left|D^{k} f(z)\right| \leq d_{1}\left(\alpha d_{2}\right)^{k-1} p\left(\alpha d_{2}|z|\right), k \geq 1$;

(iv) $T_{\alpha}(D)$ is a normal family in $|z|<t<r / \alpha d_{2}$ for all $t$ satisfying $0<t<r / \alpha d_{2}$.

We omit the proof.

\section{References}

[1] R.P. Boas, Entire functions (Academic Press, New York and London, 1954).

[2] R.P. Boas and R.C. Buck, Polynomial expansions of conalytic functions (Ergebnisse der Mathematik und ihrer Grenzgebiete, 19. Springer-Verlag, Berlin, Heidelberg, New York, 1964).

[3] J.D. Buckholtz and J.L. Frank, "Whittaker constants", Proc. London Math. Soc. (3) 23 (1971), 248-270.

[4] J.D. Buckholtz and J.L. Frank, "Whittaker constants - II", J. Approx. Theory 10 (1974), 112-122.

[5] J.D. Buckholtz and S.M. Shah, "Analytic functions with constraints on coefficients", J. Nonlinear Anal. 5 (1981), 553-564.

[6] D.M. Campbe 11, "Locally univalent functions with locally univalent derivatives", Trans. Amer. Math. Soc. 162 (1971), 395-409.

[7] A.O. Gelfond and A.F. Leontev, "On a generalization of Fourier series", Mat. Sb. (N.S.) 29 (71), (1951), 477-500.

[8] W.K. Hayman, "Coefficient problems for univalent functions and related function classes", J. London Math. Soc. 40 (1965), $385-406$.

[9] Yu.A. Kazmin, "On an interpolation problem, I", Sibirsk. Mat. Z. $(1967), 293-312$.

[10] Chi-Mun Li, "The effect of the amplitudes of coefficients of holomorphis functions in the unit circle upon the schlichtness of the functions", Chinese Math. Acta 5 (1964), 396-408. 
[11] L. Nachbin, "An extension of the notion of integral functions of the finite exponential type", Arias Acad. Brasiz Ciencias 16 (1944), $143-147$.

[12] Ch. Pommerenke, "Linear-invariante Familien Analytischer Funktionen, I", Math. Ann. 155 (1964), 108-154.

[13] Ch. Pommerenke, Univalent functions (Vandenhoeck and Ruprecht, Gottingen, 1975).

[14] Yuan-Xun Qin, "On the arguments of the coefficients in the expansion of a univalent function", Acta Math. Sinica 4 (1954), 81-86.

[15] S.M. Shah, "On the lower order of integral functions", Buzz. Amer. Math. Soc. 52 (1946), 1046-1052.

[16] S.M. Shah, "On the coefficients of an entire series of finite order", J. London Math. Soc. 26 (1951), 45-46.

[17] S.M. Shah and S.Y. Trimble, "Univalent functions with univalent derivatives", Bull. Amer. Math. Soc. 75 (1969), 153-157.

[18] S.M. Shah and S.Y. Trimble, "Univalent functions with univalent derivatives, II", Trans. Amer. Math. Soc. 144 (1969), 313-320.

[19] S.M. Shah and S.Y. Trimble, "Univalent functions with univalent derivatives, III", J. Math. Mech. 19 (1969), $451-460$.

[20] S.M. Shah and S.Y. Trimble, "Univalence of derivatives of functions defined by gap power series", J. London Math. Soc. (2) 9 (1975), 501-512.

[21] S.M. Shah and S.Y. Trimble, "Univalence of derivatives of functions defined by gap power series, II", J. Math. Anal. Appl. 56 (1976), 28-40.

[22] S.M. Shah and S.Y. Trimble, "Analytic functions with univalent derivatives", Indian J. Math. 20 (1978), 265-299. 
[23] G.S. Srivastava and O.P. Juneja, "The maximum term of a power series", J. Math. Anal. Appl. 81 (1981), 1-7.

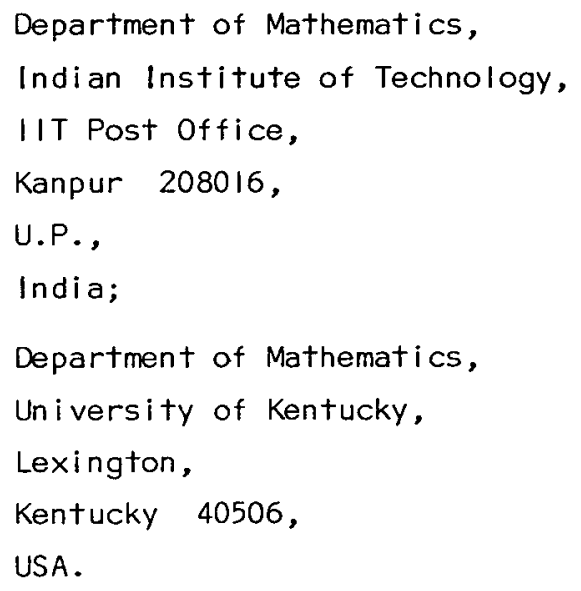

